\title{
Life-Cycle Maintenance Management Strategies for Bridges in Kuwait
}

\author{
Hesham Almomani ${ }^{1 *}$, Omar Naser Almutairi ${ }^{2}$ \\ ${ }^{1}$ Department of Industrial Engineering, Faculty of Engineering, the Hashemite University \\ ${ }^{2}$ Ministry of Kuwait Municipality, Civil Engineer, Kuwait
}

Received: 06/01/2020

Accepted: 13/04/2020

Published: 20/09/2020

\begin{abstract}
To meet the requirements of $21^{\text {st }}$ century, Kuwait ministry of public works have realized that they should use modern technologies and management system to face challenges of modern world. So, they maintenance Administration has launched a massive program which helps to meet development needs and helps to maintain the infrastructure's sustainable growth. Thus, it is very necessary to develop appropriate bridge maintenance strategies to meet serviceability criteria and life cycle cost optimization of new bridges. And main purpose was to meet the requirements of development needs. Bridge management system (BMS) is a software or we can say a management tool which helps us to maintain or manage the bridges. These are the following main points which helps decision makers to make a best decision. Defines bridge condition, identifies bridge needs for like if we need replacement of any material or renovation can be identified by BMS, cost of bridge during maintenance and management of the bridge, identifies bridges for Replacement and rehabilitation programs (MR \& R) and also identifies for the assessment of bridges. According to replacement and rehabilitation programs minor changes or maintenance of the bridges is done by Work management system (WBS). This paper proposes a framework that can enhance the effectiveness of Kuwait's Integrated Maintenance Management System (KIMMS). A survey for bridge contractors around the world was used as the research method for this study. This contains questions regarding their current bridge management system (BMS) and what are the input and output parameters it considers in the process. BIM (Bridge information modeling) technology is used to reduce the errors in designing and construction of the bridges by using BMS, BRIDGE OWNER may have a sustainability in the maintenance and enhancement of its bridge. We can determine the cash flow to be used in future for the replacement and repairment of bridge. It helps to adopt maintenance program to maintain life-cycle costs for the community. Helps to review the replacement and rehabilitation strategies. Helps to improve the economic life of infrastructure assets. After evaluating the BMSs used by the respondents, it turned out that the most effective procedure should have three main processes to be followed; maintenance planning, maintenance optimization, and maintenance decision support. Overall, the conceptualized framework considers an optimized procedure for maintenance management considering the balance in cost and quality of the process.
\end{abstract}

Keywords: Bridge Management System; Engineering Constraints; Kuwait's Integrated Maintenance Management System; Sustainability, serviceability, Bridge information model

\section{Introduction}

Within the country of Kuwait, there are 3 notable highway bridges which are considered part of the history, wherein Sheikh Jaber Al-Ahmad Al-Sabah Causeway is considered one of the longest bridges in the world spanning for about 48.5 kilometers (Naser, 2019; Ahmad \& Ahmad, 2018). There are also approximately 150 unnamed short spans to medium span highway bridges and 540 pedestrian bridges. These structures, especially the highway bridges, sustain a large volume of vehicular traffic considering that the population continues to grow. These structures are vital to maintaining the mobility and transportation of goods and services of the people, and they also provide strategic connections within the different areas of the country. As the country's governmental agency for the management of roads and highways, the Ministry of Public Works and the Public Authority for Roads \& Transportation are those that responsible for the conceptualization, design, construction, operation, and maintenance of roads and transportation systems.

Even though bridge stock in Kuwait is quite new in comparison with the stock of bridge structures in Asia and the
Americas, efficient management of bridge stocks is very important for the country. These structures bring traffic volume which plays a big part in the economy, allowing populations to reach capitals and other areas through these diversional routes. However, several challenges are being faced by society and one of them is that there are still structures within densely populated areas that pose a problem of accessibility (Wong, et al. 2001). Furthermore, there is a scarcity of materials for retrofitting and repair of these structures in some regions. Thus, maintenance management of bridges is necessary in order to provide a sustainable level of functionality and serviceability of the structure in its entire life span. Coherent and well-structured techniques and procedures for continuous and cyclical maintenance are very important.

In summary, highway bridges in Kuwait can be differentiated into two classifications. The first one considers long-span bridges (LSB) like Sheikh Jaber Al-Ahmad AlSabah Causeway for example, and these can be either suspension bridge or cable-stayed bridges. Most of these are built with modern technology equipped with monitoring systems that can see the current structural behavior of the

Corresponding author: Hesham Almomani, Department of Industrial Engineering, Faculty of Engineering, The Hashemite University, E-mail: oalmut220@gmail.com 
bridge. The second classification is composed of the short span or medium highway bridges (SMB). Some of them created in the recent decade until today are made using prestressed concrete, while those that are 25 years ago and further are made of reinforced concrete. Considering that both stated concrete configurations are prone to deterioration especially when exposed to changing humidity and weather, the maintenance management system would enhance the functionality of the structure for extended years. In this paper, the conceptualized preliminary framework for the strategic maintenance management of bridges is focused on SSMB bridges in Kuwait since the maintenance for long-span bridges is most likely well studied during the design phase.

\section{Review of Related Literature and Studies \\ 2.1 Review of Existing Bridge Management Systems}

In order to have a benchmark about the condition of present Bridge Management Systems, the result of research conducted by Yang et. al. (2006) regarding BMS in some chosen countries was utilized. The review is mainly based on a questionnaire survey form wherein most of the questions came from a 1999 Questionnaire. These questions were part of the technical deliverables of a bridge project known as "BRIME" in Europe (FEHRL, 1999). Listed below is the scope of the questionnaire form: (a) bridge management system description, (b) database information, (c) bridge condition, (d) other necessary information, (e) forecast on condition of the bridge, (f) cost analysis, (g) allocation and other details for maintenance, (h) quality control, and (i) information on administration. The survey forms were priorly delivered to 28 bridge private contractors and government administration departments in selected regions and states in countries within America, Eurasia, and Oceania. Among these target respondents, only 7 were able to submit their answers in the given deadline. Code names through the use of alphabet letters were used in order to hide the company name and keep them confidential information.

\subsection{Analysis of the Survey Responses}

The following tables 2, 3, and 4 gives a summary of the results of the survey. The tables show the general attributes, functions, components, and other information of the BMS's of the respondents. In this, the analysis and evaluation of the responses are focused on the general information of the bridge maintenance management utilized by the respondents. More than half of the contractors who replied to the survey have used BMS's for more than 10 years, considering that the latest version of BMS was provided in the year 2000. All of the respondents use technologically integrated BMS with automatic production of the database. BMS is usually utilized for bridges that are considered to be national connections. Almost all the respondents go through life cycle cost analysis using their BMS. Lastly, most of the BMSs consist of maintenance management tools for systematic inspection, planning, evaluation, and prioritization. Based on the results, the majority of the respondents utilize their BMS to discern various maintenance and repair works. For the respondent (B), the decision is mainly focused on the conditions of the components of the bridge. For respondent $\mathrm{C}$, their maintenance process depends on the available budget, and also the amount and current condition of the bridge components. For respondent $\mathrm{E}$, the computations for present economic costs are utilized in order to produce a detailed set of inspections.

Moreover, most of the respondents have a certain tool that can determine and predict the necessity for the maintenance of a bridge structure. Respondent A has a database of recorded defects from which the maintenance work for existing bridges can be decided. Respondent B considers the provided risk assessment and present condition of the bridge. Respondent $\mathrm{C}$ has an established framework in general to determine the current condition rating of the bridge. Respondent D has developed its IT-related techniques for conceptualization and prioritization of maintenance works. Respondent E, they have provided a set of modules that can be used to determine the classification and condition of the damage based on the inspection report, which can then be used to create a strategic prototype of the optimal strategy for maintenance works. Respondent $\mathrm{F}$ decides based on their inhouse maintenance programming. Furthermore, to determine the best option for maintenance management, respondent A utilizes value management based on the results of risk assessments. Respondent B uses their experience and also utilizes the 'Strengthening Guidelines Manual'. Respondent C determines the best maintenance management option by balancing the budget and also the professional engineering preferences. Respondent $\mathrm{E}$ decides on the lowest cost of the provided set of alternatives. Respondent F most of the time combines the result of site investigation, engineering perception, and cost analysis to select the most suitable maintenance strategy (Ahmad \& Sahar, 2019). In many countries, ranges of condition ratings for bridges are used to recommend maintenance strategy. For respondent $\mathrm{B}$, preliminary information comes from the inspector who also initially recommends what he thinks is the best treatment option. For respondent $\mathrm{C}$, specific activities are done during inspection to determine such maintenance needs. The output reports from the inspection are then reviewed by the head which then draws the plan of maintenance works to be done. Most of the BMS included in this study does not have a function that can generate the most cost-efficient maintenance strategy which considers different constraints on the condition of the bridges. However, most of the respondents have BMSs that can produce a strategy for maintenance prioritization based on some design parameters such as traffic vehicle volume, the significance of freight route, analysis of risks, etc.

Table 1: General Information on the Bridge Management Systems of the Respondents

\begin{tabular}{|l|c|c|c|c|c|c|c|}
\hline \multirow{2}{*}{ Questions } & \multicolumn{7}{|c|}{ Responses of the Respondents } \\
\cline { 2 - 8 } & A & B & C & D & E & F & G \\
\hline What is the bridge classification within the BMS? & SMB & Both & Both & Both & Both & Both & SMB \\
\hline Is it a digital BMS? & Y & Y & Y & Y & Y & Y & Y \\
\hline How long in years has the BMS been used? & 6 & 9 & $>10$ & 15 & 19 & $>15$ & 5 \\
\hline Is it connected to the road management system? & N & Y & Y & N & N & Y & Y \\
\hline How many bridges are there in the Database? & 9000 & 3000 & 12000 & 4600 & 30000 & 3000 & 896 \\
\hline Does it include special documentation for BMS? & $\mathrm{Y}$ & $\mathrm{Y}$ & $\mathrm{Y}$ & $\mathrm{Y}$ & $\mathrm{Y}$ & $\mathrm{Y}$ & $\mathrm{Y}$ \\
\hline Is there a Life Cycle Cost Analysis? & $\mathrm{Y}$ & $\mathrm{Y}$ & $\mathrm{N}$ & $\mathrm{Y}$ & $\mathrm{Y}$ & $\mathrm{Y}$ & $\mathrm{N}$ \\
\hline
\end{tabular}


Table 2: Functions and Components of the Bridge Management Systems of the Respondents

\begin{tabular}{|c|l|}
\hline & $\begin{array}{l}\text { As recording database for structure stock inventory, capacity, and condition; } \\
\text { Conceptualization and procedure for maintenance works; } \\
\text { Scheduling for site inspections; } \\
\text { Documentation of Defects. }\end{array}$ \\
\hline \multirow{3}{*}{ B } & $\begin{array}{l}\text { Database for bridge location, structural type, and dimensions; } \\
\text { Data regarding current condition and upcoming inspection; } \\
\text { Detailed cost data; } \\
\text { Database for reports and correspondence; } \\
\text { Prioritization and scheduling or repair works. }\end{array}$ \\
\hline & $\begin{array}{l}\text { Module for inspection; } \\
\text { Module for Programming; } \\
\text { Module for Preservation; } \\
\text { Module for Results; } \\
\text { Module for Gateway (Import and export information linked different BMS systems); } \\
\text { Module for configuration and software customization. }\end{array}$ \\
\hline $\mathrm{D}$ & $\begin{array}{l}\text { Database and inventory of bridges, including current condition and maintenance process; } \\
\text { Inspection organization; } \\
\text { Maintenance management model. }\end{array}$ \\
\hline $\mathrm{E}$ & $\begin{array}{l}\text { Overall maintenance strategy; } \\
\text { Set of activities; } \\
\text { Database of the structure; } \\
\text { Database of information; } \\
\text { Modules for procedures. }\end{array}$ \\
\hline $\mathrm{G}$ & $\begin{array}{l}\text { Database and inventory; } \\
\text { Inspection organization; } \\
\text { Evaluation and Conceptualization of Maintenance Procedure. }\end{array}$ \\
\hline & Database and inventory. \\
\hline
\end{tabular}

Table 3: Additional Information Regarding Maintenance Strategy in the Respondents' BMSs

\begin{tabular}{|c|c|c|c|c|c|c|c|}
\hline & $\mathbf{A}$ & B & C & D & $\mathbf{E}$ & $\mathbf{F}$ & $\mathbf{G}$ \\
\hline $\begin{array}{l}\text { Does the BMS utilize a certain method to determine whether to repair, } \\
\text { strengthen, or replace the components of the bridge considering that } \\
\text { it is damaged already? }\end{array}$ & $\mathrm{N}$ & $\mathrm{Y}$ & $Y$ & $\mathrm{Y}$ & $\mathrm{Y}$ & $\mathrm{N}$ & $\mathrm{N}$ \\
\hline $\begin{array}{l}\text { Does the BMS utilize a certain method in order to determine it is } \\
\text { necessary to do the maintenance work already for a specified bridge } \\
\text { aside from professional judgment? }\end{array}$ & $\mathrm{Y}$ & $\mathrm{Y}$ & $Y$ & $\mathrm{Y}$ & $\mathrm{Y}$ & $\mathrm{Y}$ & $\mathrm{N}$ \\
\hline $\begin{array}{l}\text { Does the BMS utilize a certain method in order to determine the most } \\
\text { optimal choice for maintenance procedures aside from professional } \\
\text { judgment? }\end{array}$ & $\mathrm{Y}$ & $\mathrm{Y}$ & $\mathrm{Y}$ & $\mathrm{N}$ & $\mathrm{Y}$ & $\mathrm{Y}$ & $\mathrm{N}$ \\
\hline $\begin{array}{l}\text { Does the BMS contain certain options for maintenance process } \\
\text { recommendation which depend on criteria such as type of damage, } \\
\text { level of damage, or other factors? }\end{array}$ & $\mathrm{N}$ & $\mathrm{Y}$ & $\mathrm{Y}$ & $\mathrm{N}$ & $\mathrm{N}$ & $\mathrm{N}$ & $\mathrm{N}$ \\
\hline $\begin{array}{l}\text { Does the BMS provide an optimized or cost-efficient maintenance } \\
\text { strategy considering different levels of conditions of the bridge? }\end{array}$ & $\mathrm{N}$ & $\mathrm{N}$ & $\mathrm{N}$ & $\mathrm{Y}$ & $\mathrm{Y}$ & $\mathrm{N}$ & $\mathrm{N}$ \\
\hline $\begin{array}{l}\text { Does the BMS provide a prioritized strategy for maintenance works } \\
\text { in the bridge stock considering that the budget is scarce? }\end{array}$ & $\mathrm{N}$ & $\mathrm{Y}$ & $\mathrm{Y}$ & $\mathrm{Y}$ & $\mathrm{Y}$ & $\mathrm{N}$ & $\mathrm{N}$ \\
\hline
\end{tabular}

\subsection{Review of Current Bridge Maintenance Management \\ Practice in Kuwait}

Kuwait is one of the countries that has utilized its unique bridge maintenance management system, named as Kuwait Infrastructure Maintenance Management System (KIMMS) which was completed in the year 2000. This integrated maintenance management program can be utilized to determine the current condition, forecast repair necessities and recommend optimal solutions and alternatives for various infrastructure components such as highway bridges and road pavements (Baumgardner, n.d.). One of the distinct features of this system is that it has an Integrated Priority Program (IPP) which evaluates the alternatives for maintenance strategy, and which can also give recommendations for the time interval of implementation. Moreover, it also provides a list of projects that needs to be prioritized for maintenance works. Budgeting can be done by considering groups of structural components depending on the maintenance requirements, and these are organized in the IPP to determine the most cost-efficient maintenance strategy. Data for more than 260 highway and pedestrian bridges, together with 5000-kilometer road pavements are maintained and kept in KIMMS (Karan et. al., 2001). This database is utilized by six departments for maintenance management of the Ministry of Public Works, which are divided by project budget constraints, and each of these departments is responsible for various types of structural components (Elis et. al., 2010). Although this system is very promising to have in any country, there are still disadvantages that can be found not just on the system, but on the way how the maintenance management is being implemented. One disadvantage of this system is that the chosen maintenance strategy sometimes does not fit well with the provided budget, considering that there are many factors and parameters included in the selection phase. Thus, the optimal maintenance 
planning strategy should be cost-efficient and must be within the budget. Another disadvantage is the lack of professional judgment which should come from the designated engineers. Most of the decision making is done in the system to make everything digital, setting aside the perception of the professionals which is very important for the structure (Ahmad
\& Ahmad, 2019). A good example of this aspect is that as years go by, there is newly discovered repair condition for a certain structure which is not recorded in the database. Thus, the final decision for the most applicable maintenance strategy must have both the insights of the integrated system and the recommendations of the professionals.

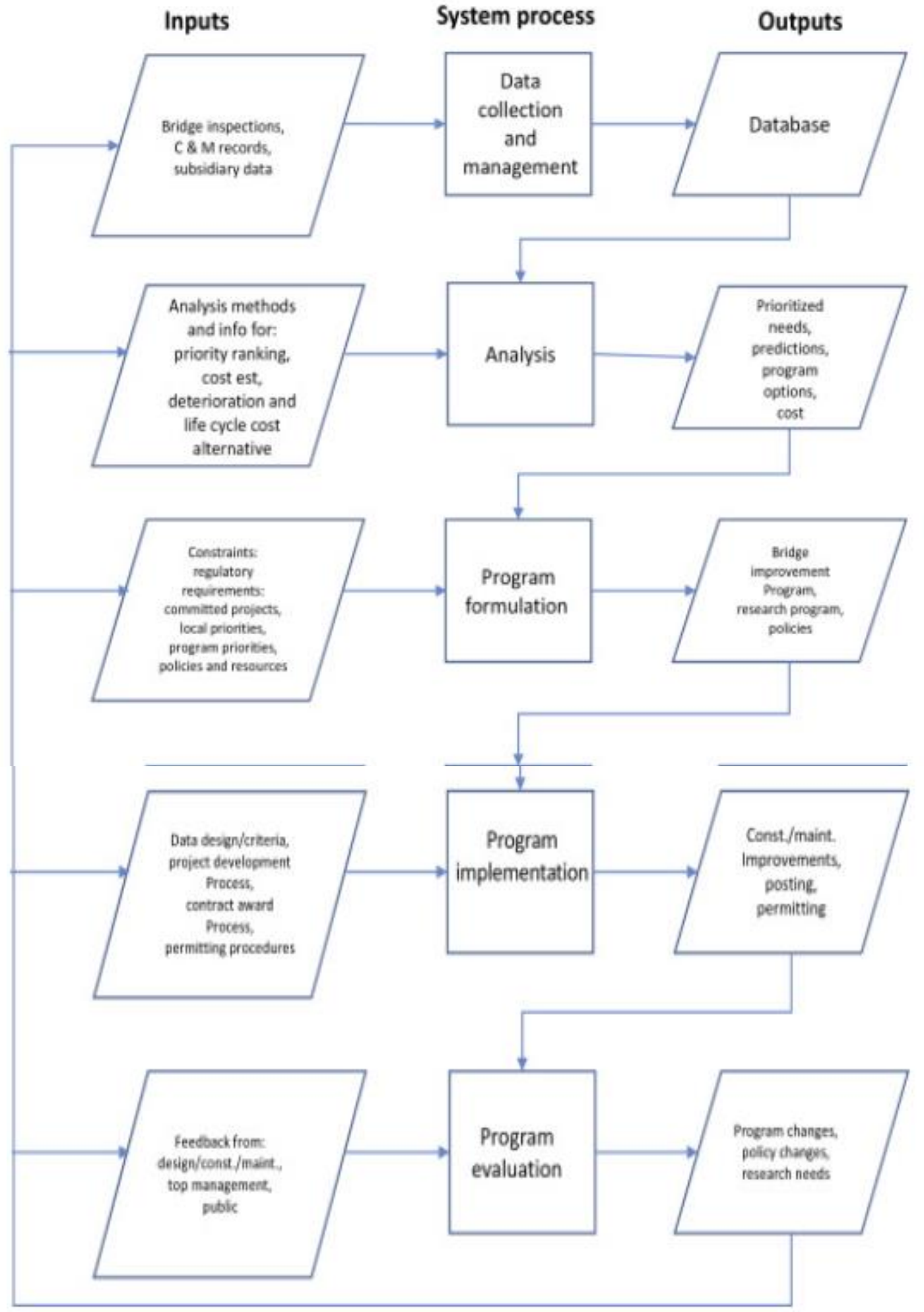

Figure 1: This is how BMS Works 


\subsection{Interim Objectives}

Based on the review of existing BMSs of the respondents which comprise of selected countries, and comparing them with Kuwait's own maintenance management system KIMMS, the following functions for bridge maintenance system can be proposed to enhance the current maintenance strategy used for the country. (a) Planning. This pertains to the conventional way of how the BMS chooses the most suitable maintenance method for such damage to a bridge component. (b) Optimization. This pertains to determining the most suitable maintenance strategy, which is at the same time considering cost analysis to have the most cost-efficient choice during the lifespan of the structure. (c) Decision support. This pertains to maintenance planning with professional judgment taking into account both the result of the BMS and professional opinions.

\section{Methodology}

\subsection{Bridge Management System}

These are the following methods by which we can do bridge maintenance and management. First of all, we are going to do discuss "Bridge management software" or BMS. Since from several decades, operations of bridges are managed by local labor. But by the 1980s, significant interest taken by bridge management system at the state and federal level. There are many functions can be done with BMS. Some of them we are going to describe here like: (a) BMS helps BRIDGE OWNER to have a sustainability in the maintenance and enhancement of its bridge; (b) determines the cash flow to be used in future for the replacement and repairment of bridge; (c) it helps to adopt maintenance program to maintain life-cycle costs for the community; (d) helps to review the replacement and rehabilitation strategies; (f) helps to improve the economic life of infrastructure assets; ( $\mathrm{g}$ ) it helps to review bridge management system which is used bridge owner's bridge assets; (h) level of service and condition can be managed very effectively. Now let's have some discussion about components of bridge management system. The most important and most effective component of bridge management system is inspection module and inventory module. For the database to be functional, it must have input in it. And this data is collected by inspection module. Similarly, some other components are here with names like maintenance module, Database module, Maintenance optimization module and Life cycle cost (LCC). These modules are used to solve issues related to them. This is how BMS Works (Figure 1).

\subsection{Proposed Framework for Enhanced KIMMS for Kuwait}

In recent years, combined mathematical and engineering methods were developed for the optimization of structural maintenance for bridges considering multiple constraints and parameters. The fundamental concept of these methods is to reduce the life-cycle cost of the structure sacrificing the serviceability and performance of the bridge structure. One good example is research conducted by Itoh and Liu (1999), wherein they utilized the technique of genetic algorithms to propose an optimized rehabilitation development plan for bridge decks which was designed based on the maintenance cost and degree of damage, simultaneously. Liu and Fangopol (2004) also researched with the same objective but with a different framework as they utilized three different objective parameters for coming up with the best tradeoff for maintenance management. These functions are safety index, condition index, and cumulative life-cycle maintenance cost. Inagaki et. al. (2005). Moreover, a strategic cycle for maintenance works was proposed Inagaki et. al. (2005) considering indicators for the current condition of the structure and renewal costs. The initial framework for the enhancement of KIMMS mainly for the short span to medium span bridges in Kuwait is proposed based on these collected principles, which focuses on providing a strategic maintenance plan that considers the life-cycle costs of the structure, without compromising the serviceability, safety, and sustainability of the bridge during its life span.

\subsection{Primary Objective of the Existing BMS in Kuwait}

For the proposed enhancement of the current BMS used in Kuwait, the following set of objectives is utilized which are based on research conducted by Wong et. al. (2001).

1. Performance: this pertains to the continuous service of bridges with the least disruption considering the codes and standards accepted in the local community.

2. Prediction: this refers to the understanding and monitoring of bridge stock with adequate information to effective and efficient maintenance planning.

3. Funding: this ensures that the budget provided by the client produces a reasonable result.

4. Social: this takes into account other responsibilities of the bridge such as sustainability and safety.

To address these primary objectives while taking into consideration the three functions provided for the enhancement of BMS in Kuwait, namely, maintenance planning, optimization, and maintenance decision support, it is appropriate to formulate suitable life-cycle strategies for maintenance management. The resulting strategies take into consideration various criteria and parameters other than the economical aspect, but also the technical aspects. The main aim of the economical aspect is to reduce the present value of the entire life-cycle cost which includes the maintenance works. The objective of the technical aspect is to make sure that the safety, serviceability, and aesthetic appearance of the bridge is not compromised. Thus, the cost objective must be wellbalanced with the expected performance and quality of the structure.

\subsection{Constraints Considered in Strategic Maintenance Management}

All the constraints considered in coming up with the best strategic way for maintenance management are discussed in this section. These constraints are mainly based on a study conducted by Yang, et. al. (2006).

\subsubsection{Technology - Safety and Serviceability}

The primary objective of maintenance management is to guarantee the safety and serviceability of the bridges in their projected life span, considering the required maintenance works and damage repairs. To reiterate the review in the previous section, Liu and Frangopol (2004) evaluated the performance of damaged bridges by utilizing indices named condition and safety. In this study, the condition index has a total of more than 350 data, with 0 to 3 as a value representing the bridges' damage condition based on visual inspection. The following are the characteristics of each numerical representation.

- 0 - no chloride contamination

- 1 - the onset of corrosion

- 2 - the onset of cracking

- 3 - loose concrete or significant delamination

There are still higher condition indices made for those damages that are worse than the enumerated conditions. The safety index pertains to the ratio of the actual to the calculated live load resistance of the bridge, which takes into account the degree of reliability of the damaged element of the bridge. However, the safety index is quite different from the condition 
index because higher safety levels describe damages that are safer.

\subsubsection{Economy - Present Value of Cumulative Life-Cycle Maintenance Cost}

The accumulated life-cycle maintenance cost is the total costs of every repair or retrofitting works done during the functionality period or service lifespan of the bridge, which are reduced to the present economic value considering the present, and this is can be presented in the equation below,

$C_{P V}=\sum_{i=1}^{N} \frac{C_{t_{i}}}{(1+v)^{t_{i}}}$

where $\mathrm{CPV}_{\mathrm{PV}}$ is the present value of the combined cost of maintenance works, $\mathrm{N}$ is the total number of maintenance periods, $\mathrm{v}$ is the discount rate, and $\mathrm{Ct}$ is the cost of maintenance work required. The optimal problem for maintenance management deals with the cost-efficient budgeting not just the maintenance cost but also the maintenance efforts so that there will be an equity and that the objectives for all the constraints described can be attained, and this problem is briefly described as follows provided on the results of the study conducted by Liu and Frangopol (2004). (a) Coming up with the smallest possible condition index during the lifespan of the bridge; (b) coming up with the largest possible safety index during the lifespan of the bridge, and; (c) coming up with the lowest possible present value of total maintenance cost during the lifespan of the bridge.

\subsubsection{Sustainability - Environmental, Social, and Economic Impact}

Economic, environment, and community are three aspects that are utilized internationally to define the extent of sustainability as a constraint. The stability of the environmental aspect along with industrial developments must be wellestablished which is the main principle of sustainability. KPIs or also known as key performance indicators are applied in modern designs to balance the environmental, social, and economical aspects of the maintenance management strategies for bridges.

\subsection{Life Cycle Cost Optimization}

Life cycle cost optimization is one of the main objectives of the framework to be proposed and it obtained based on the requirements of VFM or "value for money". VFM is defined as a parameter that circulates three E's, namely, economy, efficiency, and effectiveness (Rutter and Gidado, 2005). Each is defined as follows depending on their usage in this paper.

- The economy pertains to minimizing the total cost of raw materials and resources used for the maintenance works of the bridge.

- Efficiency means maximizing the output to input ratio of the bridge maintenance management procedure, wherein the output pertains to the performance and quality of the work, while input pertains to the life cycle cost.

- Effectiveness refers to maximizing the utility value, which is described as the welfare provided by the client to a certain project with a particular profit, as well as risk.

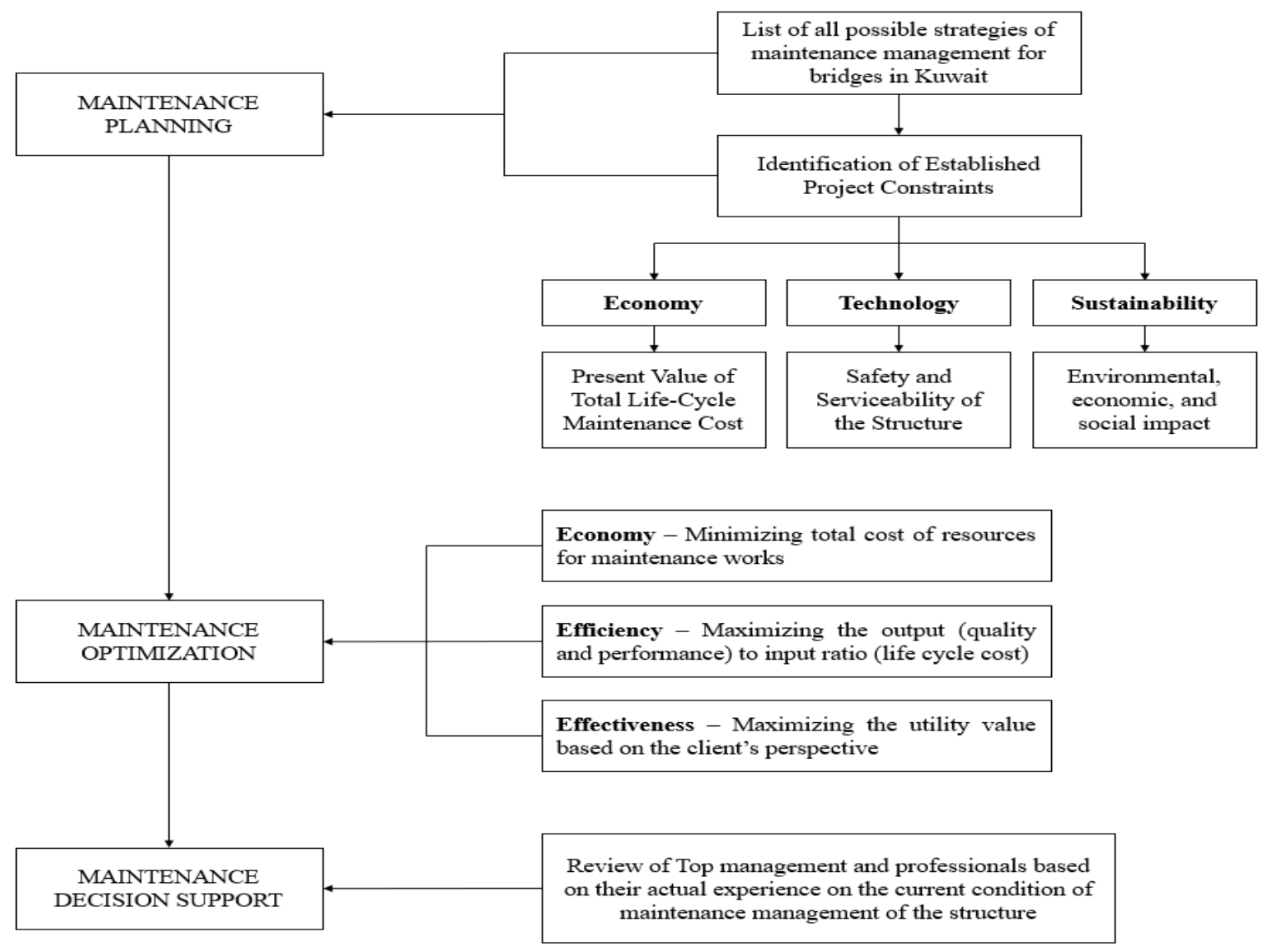

Figure 2: Proposed Framework for the Enhanced KIMMS 


\subsection{Proposed Framework}

The development of the framework for Life-cycle Maintenance Management of short span to medium span bridges in Kuwait consists of three different steps as shown in Figure 2. The summary of this procedure is listed as follows: (a) maintenance planning - determination of a ranked list of maintenance management strategies based on the current conditions of the damaged bridge component considering different alternatives; (b) maintenance optimization - the generated list of maintenance strategies are then assessed using cost analysis, which is then balanced with technological and sustainability constraints; and (c) maintenance decision support - the optimized maintenance management strategy together with proposed alternatives is then reviewed by the administration and the professionals to decide whether to stick with the optimized strategy or the select from the alternatives.

\section{Conclusions}

In this study, a proposed enhancement for KIMMS, or the current bridge maintenance management system utilized in Kuwait, is developed focusing on a balanced decision making for the maintenance management strategy by adding up some constraints for consideration such as cost optimization and decision support from professionals. This would satisfy almost all the constraints in bridge engineering design such as economic, safety, serviceability, and sustainability. The output strategy represented by the framework takes into account these general constraints without compromising the existing methods and practices utilized by the KIMMS. This proposed framework provides certain features that can identify whether the objectives and requirements of all the constraints are met with a balanced maintenance program and life-cycle cost.

\section{Ethical issue}

Authors are aware of, and comply with, best practice in publication ethics specifically with regard to authorship (avoidance of guest authorship), dual submission, manipulation of figures, competing interests and compliance with policies on research ethics. Authors adhere to publication requirements that submitted work is original and has not been published elsewhere in any language.

\section{Competing interests}

The authors declare that there is no conflict of interest that would prejudice the impartiality of this scientific work.

\section{Authors' contribution}

All authors of this study have a complete contribution for data collection, data analyses and manuscript writing.

\section{References}

Ahmad, I., \& Ahmad, S. (2019). The Mediation Effect of Strategic Planning on The Relationship Between Business Skills and Firm's Performance: Evidence from Medium Enterprises in Punjab, Pakistan. Opcion, 35(24), 746-778.

Ahmad, I., Sahar. (2019). Waste Management Analysis From Economic Environment Sustainability Perspective. International Journal Of Scientific \& Technology Research 8(12), 1540-1543.

Ahmad, I., \& Ahmad, S. (2018). Multiple Skills and Medium Enterprises' Performance in Punjab Pakistan: A Pilot Study. Journal of Social Sciences Research, 7(2010), 44-49.

Baumgardner, R. (n.d.). "Kuwait Infrastructure Maintenance Management System." Kuwait Infrastructure Maintenance Management System (KIMMS), ITX Technologies/Soor Engineering Bureau, <https://proceedings.esri.com/library/userconf/proc96/to300/pap2 76/p276.htm> (Apr. 7, 2020).
2. Ellis, R. M., Cheetham, A., Hong, K., \& Thompson, P. D. (2010, July). Integration of bridge management systems (BMS) and pavement management systems (PMS). In Bridge Maintenance, Safety, Management, and Life-Cycle Optimization: Proceedings of the Fifth International IABMAS Conference, Philadelphia, USA, 11-15 July 2010 (p. 233). CRC Press.

3. FEHRL (1999), "Deliverable D4 under the 'BRIME' project Review of existing BMS and definition of inputs for the proposed BMS", The UK's Transport Research Laboratory (TRL), Forum of European Highway Research Laboratories (FEHRL), <http://www.trl.co.uk/brime/d4.pdf> (accessed in April 2006).

4. Inagaki H., Fujino Y., Kitagawa K. and Kawamura K. (2005), "The maintenance and management strategy of bridges for local government in Japan", Proceedings of the 5th International Conference on Bridge Management, Apr. 11-13, Guildford, $377-$ 384.

5. Itoh Y. and Liu C. (1999), "Multiobjective optimization of bridge deck maintenance", Case Studies in Optimal Design and Maintenance Planning of Civil Infrastructure, 136-151.

6. Karan, M. A., Al-Kulaib, A. A., \& Haas, R. (2001, August). An Overview of Kuwait's Integrated Infrastructure Maintenance Management System (KIMMS). In Fifth International Conference on Managing Pavements Washington State Department of Transportation Foundation for Pavement Preservation International Society for Asphalt Pavements Federal Highway Administration Transportation Research Board.

7. Liu M. and Frangopol D.M. (2004), "Optimal bridge maintenance planning based on probabilistic performance prediction", Engineering Structures, 26(7), 991-1002.

8. Rutter, D., \& Gidado, K. (2005). PFI projects: their scope for delivering VFM. In The 21st Annual ARCOM Conference" $(F$ Khosrowshahi, ed.) (Vol. 1, pp. 591-9)

9. Wasmi, N. A. (2019, May 2). Kuwait inaugurates the fourth longest bridge in the world for a new megacity. Retrieved from https://www.thenational.ae/world/gcc/kuwait-inaugurates-thefourth-longest-bridge-in-the-world-for-new-megacity-1.856527

10. Wong P.C., Wong C.Y., and Darby J. (2001), "Bridge Management in Hong Kong: the selection of appropriate techniques", Current and future trends in bridge design construction and maintenance 2 : safety, economy, sustainability and aesthetics, proceedings of the international conference organized by the Institution of Civil, Apr. 25-26, Hong Kong, 3-12.

11. Yang, Y. N., Pam, H. J., Kumaraswamy, M. M., \& Ugwu, O. O. (2006, November). Life-cycle maintenance management strategies for bridges in Hong Kong. In Proceedings of the Joint International Conference on Construction Culture, Innovation and Management (pp. 26-35).

12. Mirzaei, Z., et al. The IABMAS bridge management committee overview of existing bridge management systems. Technical Report, Institute for Construction and Infrastructure Management, Swiss Federal Institute of Technology, Zurich, Switzerland, 2014

13. [https://www.bing.com/search?q=bridge+management+system+in + kuwait $\&$ form $=$ EDGTCT\&qs $=$ PF \&cvid $=21 \mathrm{e} 2 \mathrm{aa} 21793840 \mathrm{~b} 3 \mathrm{a} 88$ $1 \mathrm{dd} 789 \mathrm{e} 468 \mathrm{~d} 39 \&$ refig $=4515857 \mathrm{a} 91 \mathrm{~b} 6404 \mathrm{aeb} 4305 \mathrm{c} 9 \mathrm{bb} 9302 \mathrm{e} 0 \&$ $\mathrm{cc}=\mathrm{US} \&$ setlang=en-

US\&elv=AXK1c4IvZoNaPoPnS\%21QRLOMA2G3Tbi1h7izPnn WzDg8P0b*tVtHP1 y7F59TSUS9OdumZFAQL3Jd6feMPbHF\% 2155an8CEeZLzG3r9K1*Z6pig6\&plvar=0\# ]

[http://montreal.ciise.concordia.ca/infra/publications/Jerry/Thesis /Final\%20Submmision.pdf ] 